\title{
Chronische Quecksilberintoxikation bei Kindern aus zwei Familien durch kosmetische Anwendung einer Bleichcreme seitens der Mütter
}

\section{Chronic Mercury Intoxication in Children of Two Families Due to the Cosmetic Application of a Mercury Containing Bleaching Cream by the Mothers}

Autoren

Institute

\author{
B. Held ${ }^{1}$, C. Bayerl
}

Facharzt für Dermatologie und Allergologie, Villingen-Schwenningen

Klinik für Dermatologie und Allergologie, HSK Wilhelm Fresenius Klinik Wiesbaden

\section{Bibliografie}

DOI $10.1055 / \mathrm{s}-2008-1077492$

Akt Dermatol 2008; 34 :

274-278 @ Georg Thieme

Verlag KG Stuttgart · New York ISSN 0340-2541

Korrespondenzadresse

Prof. Dr. med.

\section{Christiane Bayerl}

Klinik für Dermatologie und Allergologie HSK, Wilhelm Fresenius Klinik Aukammallee 39 65191 Wiesbaden christiane.bayer!@ hsk-wiesbaden.de

\section{Zusammenfassung \\ $\nabla$}

Wir berichten über zwei Familien $(A: n=4, B$ : $n=5$ ) aus Kosovo-Albanien, deren Mütter Bleichcremes aus kosmetischen Gründen angewendet hatten, die von einem ländlichen Markt stammten und $12 \%$ elementares Quecksilber enthielten. Erhöhte Quecksilberspiegel im Urin waren erstmals bei einer Reihenuntersuchung in der Schule bei je einem Sohn aufgefallen. Dies führte zu einem Screening bei den anderen Familienmitgliedern.

Die Anamnese zeigte bei Familie A keine Auffälligkeiten. Bei Familie B bestanden unspezifische Symptome wie Kopfschmerzen, Abgeschlagenheit, Müdigkeit und Magenschmerzen. Beide Familien zeigten kein Feer-Syndrom (Akrodynie). Ein nephrotisches Syndrom oder eine Anämie waren durch Blutuntersuchungen ausgeschlossen worden. Eine neurologische Untersuchung, EEG und körperliche Untersuchung waren unauffällig.

\section{Einleitung}

Quecksilber und seine anorganischen und organischen Verbindungen werden nach wie vor in größerem Maßstab für industrielle Zwecke vor allem in der Elektrotechnik (Kathodenmaterial in der Chloralkalielektrolyse), aber auch in der Medizin z.B. für Zahnfüllungen, Augentropfen, Antiseptika und Dermatika eingesetzt. Als weitere Quecksilberquelle sind Umweltbelastungen durch Aufnahme organischer Quecksilberverbindungen mit der Nahrung zu nennen. Als Pflanzenschutzmittel ist es seit 1980 nicht mehr in Verwendung. Laut Gefahrstoffverordnung ist es auch als Holzschutzmittel, zur Imprägnierung von industriellen Textilien und in der Wasserauf-
Mit Massenabsorptionsspektroskopie zeigten sich im 24-Stunden-Sammelurin die höchsten Werte bei den weiblichen Familienmitgliedern, die die Bleichcreme aus kosmetischen Gründen aufgetragen hatten (Familie A, A1: 226, A2: 86, A3: 25, A4: $14 \mu \mathrm{g} / \mathrm{l}$; Familie B, B1: 363; B2: 192; B3: 61; B4: 41; B5 $29 \mu \mathrm{g} / \mathrm{l}$ ). Die Referenzwerte für Erwachsene liegen bei $<7 \mu \mathrm{g} / \mathrm{l}$ im 24-Stunden-Sammelurin. Nach Behandlung mit dem Komplexbilder DMPS (2,3 -dimercaptopropane1-sulfonate, Sodium salt, Dimaval ${ }^{\circledR}$ ) mit $5 \mathrm{mg} / \mathrm{kg}$ Körpergewicht sanken die Quecksilberspiegel unter die obere Grenze der Referenzwerte und die Symptome verschwanden.

Bei sensiblen Individuen wird ein Symptomenkomplex (Mikromercuralismus) beschrieben mit sporadischer Müdigkeit, Abgeschlagenheit, gastrointestinalen Beschwerden, Intentionstremor (Tremor mercuralis) ab einer Konzentration von $100 \mu \mathrm{g} / \mathrm{l}$ Quecksilber im Urin und $35 \mu \mathrm{g} / \mathrm{l}$ im Blut Erwachsener. In beiden Familien muss die Aufnahme des Quecksilbers bei den Jungen durch die Haut erfolgt sein, durch Einatmen oder Nahrungsaufnahme.

bereitung verboten $[1,16]$. Auf der japanischen Insel Kyushu kam es im kleinen Küstenort Minamata während der 50er-Jahre zu Massenvergiftungen, als eine Kunststofffabrik belastete Abfälle einleitete. Die Bevölkerung ernährte sich vom Fischbestand aus diesen Gewässern. 1971 kam es im Irak zu Todesfällen nach Genuss von Brot aus Weizen, das mit einem Methylquecksilberhaltigem Fungizid gebeizt worden war.

Elementares, anorganisches Quecksilber ist bei Raumtemperatur flüssig und verdampft, ohne farblich sichtbar zu werden oder durch den Geruch aufzufallen. Die Aufnahme von anorganischem, metallischem Quecksilber erfolgt im Wesentlichen über 3 Wege. Eingeatmet werden Quecksilberdämpfe zu $80 \%$ in den Alveolen re- 


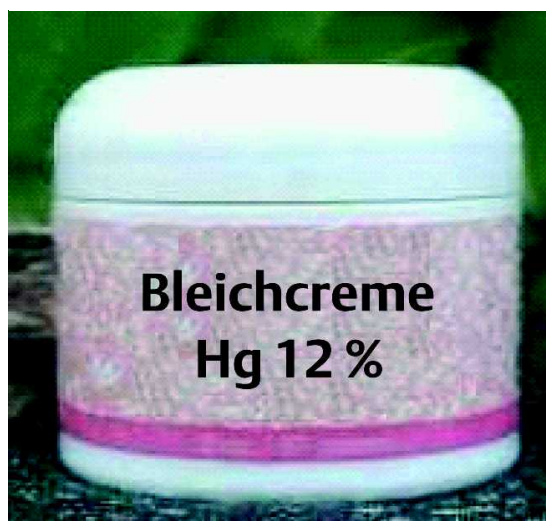

Abb. 1 Zwei Familien aus Kosovo-Albanien wendeten täglich die Haut-Bleichcreme an, bei der eine Konzentration von $12 \%$ elementarem Quecksilber gemessen wurde.

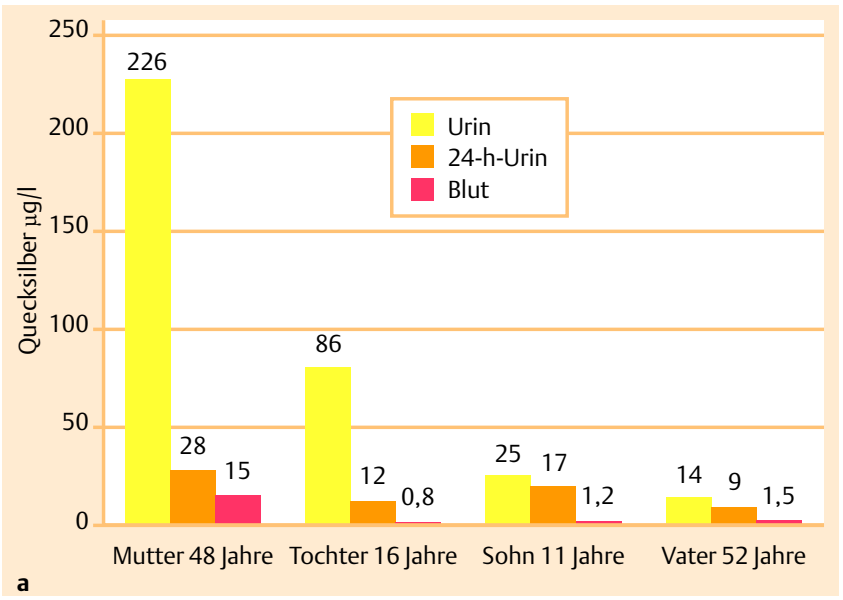

sorbiert. Bei oraler Aufnahme werden bis zu 10\% im GI-Trakt aufgenommen. Bei Hautkontakt kommt es hingegen zu einer inkonstanten Resorption. Aufgrund guter Membrangängigkeit sowie Lipophilität wird Quecksilber vor allem im ZNS, Leber und Nieren eingelagert $[2,11,16,18]$.

Akute Vergiftungen, vor allem bei Inhalation von Quecksilberdämpfen, sind charakterisiert durch Fieber, Bauchschmerzen, Diarrhoe, Erbrechen, Gingivaschwellung sowie pulmonale Toxizität, welche letztlich zu einem „acute respiratory distress syndrome“ (ARDS-Syndrom) und Tod durch progressive Hypoxie führen kann.

Chronische Quecksilberintoxikationen entstehen schleichend und führen $\mathrm{zu}$ einem als Mikromerkuralismus bezeichnetem Symptomenkomplex mit Auftreten unspezifischer Symptome wie allgemeine Schwäche, Müdigkeit, Abgeschlagenheit, Gewichtsverlust, gastrointestinale Störungen, Speichelfluss, Proteinurie, Parästhesien, Ataxie, Sprachschwierigkeiten, Hörverlusten und andere mehr. Als Tremor mercuralis wird ein Intentions-Tremor bezeichnet. Der Begriff Erethrismus mercuralis umfasst psychische Veränderungen wie Reizbarkeit, Schlafstörungen und Gedächtnisverlust.

Eine dermatologische Rarität ist das Selter-Swift-Feer-Syndrom (Akrodynie). Es umfasst Micromercuralismus-Symptome, Erethrismus mercuralis, Tremor mercuralis und akral betonte Hautveränderungen mit lividen Erythemen sowie gelegentlich Nagelveränderungen $[4,5,9,11,17]$.

Mit dem Auftreten akuter Quecksilbervergiftungen muss ab einer Konzentration von $200 \mu \mathrm{g} / 1$ 24-Stunden-Sammelurin und $50 \mu \mathrm{g} / \mathrm{l}$ Blut gerechnet werden. In Fallbeschreibungen ist das Auftreten von Mikromercuralismus-Symptomen, Tremor mercuralis und Erethrismus mercuralis bei Erwachsenen bei Queck-

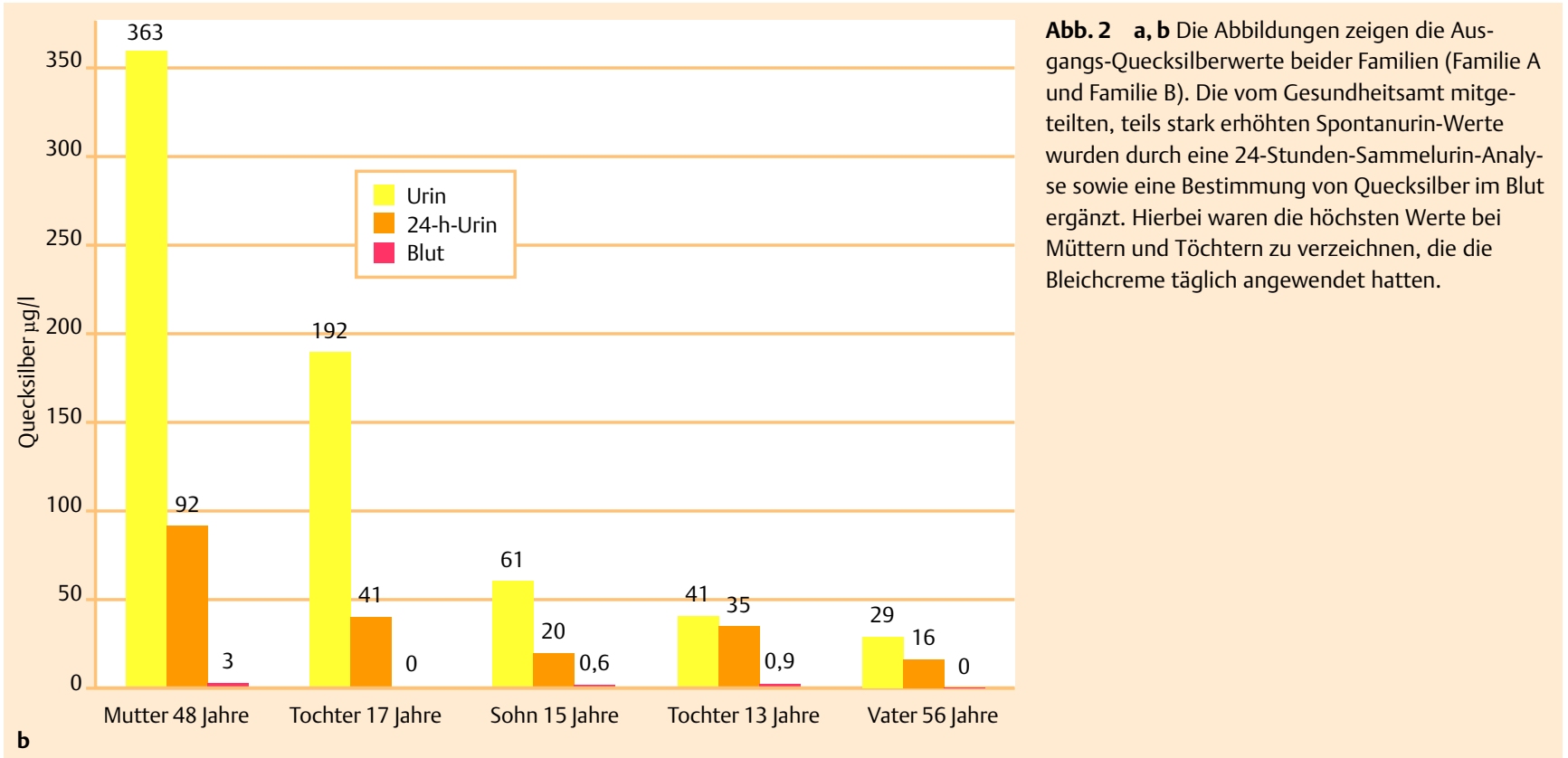


silberkonzentrationen ab $100 \mu \mathrm{g} / \mathrm{l}$ Urin und $35 \mu \mathrm{g} / \mathrm{l}$ Blut beschrieben. Man geht von einer 3-4-fach höheren Empfindlichkeit bei Kindern aus [18]. In der Literatur werden unterschiedliche Empfehlungen zur Notwendigkeit einer Therapie bei Werten ab 15 bis $30 \mu \mathrm{g} / \mathrm{l}$ 24-Stunden-Sammelurin angegeben. Die Referenzwerte für nicht arbeitsplatzexponierte Personen sind $<7 \mu \mathrm{g} / \mathrm{l}$ 24-Stunden-Sammelurin und $<4 \mu \mathrm{g} / \mathrm{l}$ Blut. Die maximal tolerierten Konzentrationen für Menschen, die am Arbeitsplatz quecksilberexponiert sind, wurden in den letzten Jahren kontinuierlich abgesenkt und belaufen sich auf weniger als $100 \mu \mathrm{g} / \mathrm{l}$ im Urin und weniger als $25 \mu \mathrm{g} / \mathrm{l}$ Quecksilber im Blut $[2,3,9,16]$. Vergiftungen und Nebenwirkungen von Bleichcremes werden von asiatischen und südostasiatischen Ländern beschrieben. Meist sind es anorganische Quecksilbersalze (Quecksilberamidchlorid, gelegentlich auch Calomel) oder metallisches Quecksilber, das sich in kosmetischen Zubereitungen findet, typischerweise in Bleichsalben oder Schönheitscremes [6,10,12,14,15]. Wir berichten über Vergiftungen, die Symptome bei mehreren Familienmitgliedern auslösten, auch bei denen, die keinen direkten Kontakt mit Bleichcremes hatten.

\section{Fallbericht}

$\nabla$

Wir berichten über zwei Familien aus Kosovo-Albanien, die vor einigen Jahren nach Deutschland emigriert waren. Erhöhte Quecksilberwerte im Spontanurin waren zunächst im Rahmen einer Reihenuntersuchung an Schulkindern, die durch das Gesundheitsamt Mannheim durchgeführt wurde, bei jeweils einem Sohn aus jeder Familie festgestellt worden.

Die hieraufhin veranlassten Spontanurin-Analysen aller Familienmitglieder ergaben stark erhöhte Quecksilberkonzentrationen bei den Familienmüttern und älteren Töchtern und erhöhte Wert bei allen anderen Familienmitgliedern.

Anamnestisch war bei Müttern und Töchtern die tägliche Anwendung einer grauen, auf albanischen Märkten frei erhältlichen und von den Händlern selbst zubereiteten Bleichcreme für kosmetische Zwecke eruierbar. Eine Analyse der Creme ergab einen Gehalt von $12 \%$ anorganischem Quecksilber, wie mit Massenspektroskopie gemessen wurde. Die Familien bewahrten die Cremedose ( Abb. 1) im Kühlschrank auf. Die Mahlzeiten wurden von den Müttern zubreitet, die hierzu keine Handschuhe trugen.

Während die weitere Anamnese bei Familie A keine Auffälligkeiten erbrachte, waren in Familie B Micromercuralismus-Symptome wie chronische Kopfschmerzen (Tochter, 17 Jahre), Bluthochdruck und Gewichtsverlust (Mutter), Konzentrationsstörungen, myozervikale Automatismen, rezidivierende Bauchschmerzen (Sohn 15 Jahre) zu verzeichnen. Bei beiden Familien waren keine Hautveränderungen im Sinne eines Selter-Swift-Feer-Syndroms vorhanden. Die durchgeführten Laboruntersuchungen mit Blutbild und Nierenwerten sowie die körperliche und neurologische Untersuchung einschließlich EEG waren ebenfalls bei beiden Familien unauffällig. Die $\bullet$ Abb. 2 a, b zeigen die Quecksilberwerte beider Familien, als die Symptomatik auffiel.

Die vom Gesundheitsamt mitgeteilten, teils stark erhöhten Spontanurin-Werte wurden durch eine 24-Stunden-Sammelurin-Analyse sowie eine Bestimmung von Quecksilber im Blut ergänzt. Hierbei waren die höchsten Werte bei Müttern und Töchtern zu verzeichnen, die die Bleichreme täglich angewendet hatten. Die Blutanalyse auf Quecksilber überschritt die Referenzwerte bei einer Person. Die Analyse des 24-Stunden-Sam-

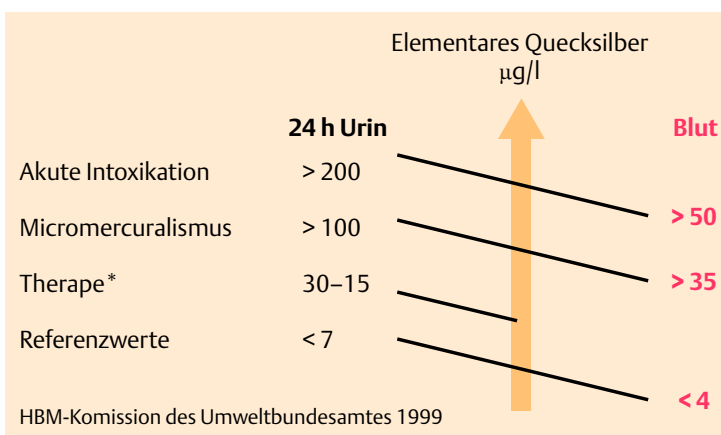

Abb. 3 Quecksilberwerte und behandlungsbedürftige Werte im 24-Stunden-Sammelurin und im Vollblut. In der Literatur werden unterschiedliche Empfehlungen zur Therapie bei Werten ab 15 bis $30 \mu \mathrm{g} / \mathrm{lm}$ 24-Stunden-Sammelurin angegeben.

\begin{tabular}{|c|c|c|}
\hline $\mathbf{a}$ & $\begin{array}{ll}\mathrm{CH}_{2}-\mathrm{CH}-\mathrm{CH}_{2}-\mathrm{SO}_{3}{ }^{-}-\mathrm{NA}^{+} \\
\mathrm{SH}_{\mathrm{SH}} & \mathrm{I}\end{array}$ & $\begin{array}{l}\text { Abb. } 4 \text { a,b Der Chelat- } \\
\text { bildner Dimaval }{ }^{\circledR} \text { (DMPS) } \\
\text { bindet Quecksilber, das in }\end{array}$ \\
\hline b & 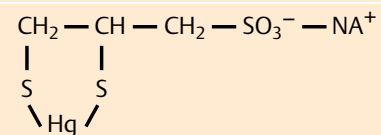 & $\begin{array}{l}\text { der Folge renal ausge- } \\
\text { schieden wird. }\end{array}$ \\
\hline
\end{tabular}

melurins brachte Quecksilberspiegel, die über den Referenzwerten lagen, und in einer Höhe, bei der eine Therapie dringend angezeigt ist ( $\bullet$ Abb. 3).

Aufgrund der hohen Werte bei Müttern und Töchtern und Bestehen von Symptomen vor allem bei den schulpflichtigen Kindern wurde eine komplexierende Therapie mit dem Chelatbildner Dimaval $^{\circledR}$ eingeleitet $(\bullet$ Abb. 4 a, b).

Nach den Empfehlungen wurde die DMPS-Therapie mit $5 \mathrm{mg} / \mathrm{kg} /$ KG begonnen und 7 Tage lang oral gegeben. Die DMPS-Therapie brachte ein deutliches Absinken sowohl der Quecksilberwerte im Urin als auch der Blutwerte bis in den Referenzbereich ( Abb.5a,b). Anamnestisch waren einige der zuvor bestehenden klinischen Symptome zu diesem Zeitpunkt bereits gebessert. Unter der DMPS-Therapie zeigten sich keine Nebenwirkungen oder Laborauffälligkeiten.

\section{Diskussion}

$\nabla$

In beiden Familien zeigten sich im Urin erhöhte Quecksilberwerte, obwohl nur die Mütter die Creme aufgetragen hatten. Ein möglicher Weg der Vergiftung für die Familienmitglieder, die keine Bleichcreme verwendet hatten, kann das Verdampfen des Quecksilbers aus der Creme und die Aufnahme über den Gastrointestinaltrakt sein, da die Mütter die Mahlzeiten für die ganze Familie vorbereitet hatten und die Creme im Kühlschrank zusammen mit Nahrungsmitteln aufbewahrt wurde. Da die Symptome einer chronischen Quecksilberintoxikation oft unspezifisch sind, sollte bei unklaren Beschwerden vor allem bei Patienten aus dieser geografischen Region an die Verdachtsdiagnose Quecksilberintoxikation gedacht werden. Auch in Mexiko und in den Südstaaten der USA ist eine „Creme de Belleza-Manning“ auf dem Markt, die 8\% Quecksilber als Calomel enthält. Darüber hinaus muss beachtet werden, dass nicht-medizinische, kosmetische Produkte eine Quelle für elementares Quecksilber darstellen, auch wenn kein direkter Kontakt mit dem Produkt besteht $[1,4,6,10,12,14,15]$. 


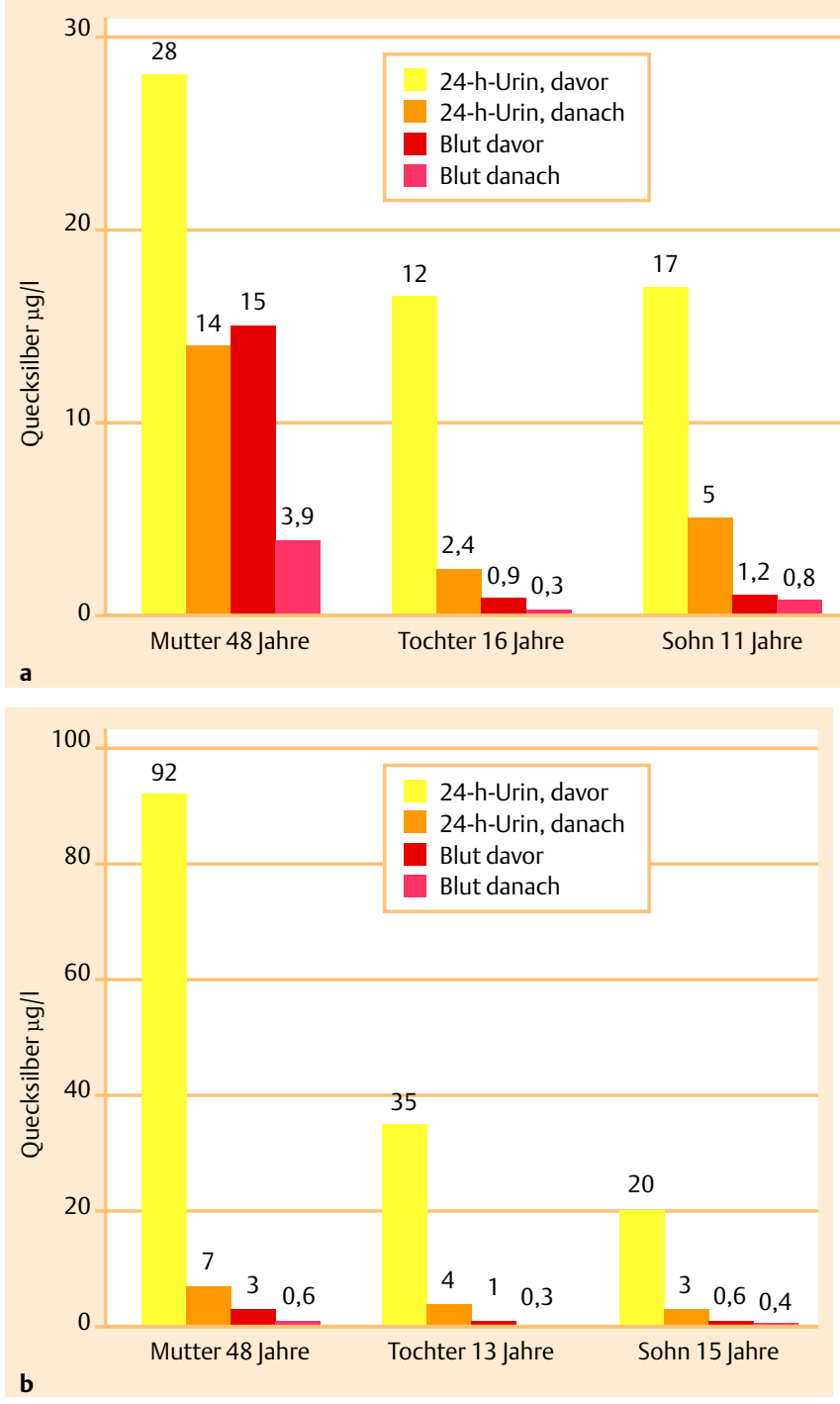

Für das Screening im Verdachtsfall ist die Spontanurin-Analyse ausreichend, bei auffälligen Werten und zur Therapieentscheidung muss eine Bestätigung über 24-Stunden-Sammelurin erfolgen. Der Quecksilberwert im Urin spiegelt nicht die Höhe des mit der Nahrung aufgenommenen Quecksilbers wider, z.B. in Fisch. Für die Messung niedriger Konzentrationen anorganischen Quecksilbers ist der 24-Stunden-Sammelurin eine praktikable und sensitive Methode des Biomonitoring [13].

DMPS ist ein Komplexbildner zur Behandlung akuter und chronischer Intoxikationen mit Quecksilber, Blei, Arsen und Kupfer. Es findet dann eine chemische Bindung zwischen DMPS und Quecksilber über Sulfurgruppen statt und dieser Komplex wird über den Urin ausgeschieden [7,8]. Eine Kontrolle der Quecksilberwerte 24 Stunden nach der DMPS-Therapie im Urin und im Blut zeigte deutlich reduzierte Quecksilberspiegel, die im Referenzbereich lagen. Auch die klinischen Beschwerden bei Familie B endeten nach der ausleitenden Therapie. Die Behandlung mit DMPS in der Therapie chronischer Quecksilberintoxikationen war bei den von uns behandelten Fällen effektiv und wurde ohne Nebenwirkungen auch von Kindern gut vertragen.
Abb. 5 a ,b Vergleich der im 24-Stunden-Sammelurin und Blut bestimmten Quecksilberwerte vor Therapiebeginn und 4 Wochen nach Abschluss einer 14-tägigen Dimaval ${ }^{\circledR}$-Therapie bei Familie A und Familie B.

\section{Abstract}

\section{Chronic Mercury Intoxication in Children of Two Families Due to the Cosmetic Application of a Mercury Containing Bleaching Cream by the Mothers $\nabla$}

We report about two families ( $A$ : $n=4, B: n=5$ ) from the KosovoAlbanian region where the mothers had been using a skin bleaching cream for cosmetic reasons from a rural market containing $12 \%$ elementary mercury. Elevated mercury levels in urine had first been detected in a survey of school pupils in one son of each family; this led to screening of all family members. Medical history showed no abnormalities in family A. In family B unspecific symptoms occurred such as headache, fatigue and bowel pain. In both families, no symptoms of Feer-syndrome (acrodynia) were observed. Nephrotic syndrome or anemia were excluded by blood analyses. Neurological examination, EEG and physical examination were normal.

Using flameless atomic absorption spectroscometry, $24 \mathrm{~h}$ urine samples revealed elevated mercury levels with highest values in those female family members who had used the cream for cosmetic purposes (family A, A1: 226, A2: 86, A3: 25, A4: $14 \mu \mathrm{g} / \mathrm{l}$; family B, B1: 363; B2: 192; B3: 61; B4: 41; B5: $29 \mu \mathrm{g} / \mathrm{l}$ ). Reference values for adults are $<7 \mu \mathrm{g} / \mathrm{l}$ in $24 \mathrm{~h}$ urine. After antidote treatment with DMPS (2,3 -dimercaptopropane-1-sulfonate, Sodium salt, Dimaval ${ }^{\circledR}$ ) with $5 \mathrm{mg} / \mathrm{kg}$ bodyweight for 7 days, biomonitoring revealed a mercury level lower than the reference value and the symptoms ceased.

In sensitive individuals, a symptom-complex (micromercuralism) with sporadic weakness, fatigue, gastrointestinal disorders, intention tremor (tremor mercuralis), alterations of the personality and memory loss (Erethrismus mercuralis) occur at a concentration of $100 \mu \mathrm{g} / \mathrm{l}$ mercury in urine and $35 \mu \mathrm{g} / \mathrm{l}$ in blood in adults. In the two families, absorption of the mercury in the boys might have occurred through the skin, by breathing or by ingestion. 


\section{Literatur}

1 Aberer W, Gerstner G, Pehamberger H. Ammoniated mercury ointment: outdated but still in use. Contact Dermatitis 1990; 23: 168-171

2 Anonym. Stoffmonographie Quecksilber - Referenz- und Human-Biomonitoring-(HBM)-Werte. Bundesgesundheitsbl-GesundheitsforschGesundheitsschutz 1999; 42: 522 - 532

3 Anonym. Aktualisierung der Referenzwerte für Blei, Cadmium und Quecksilber im Blut und Urin von Erwachsenen. Stellungnahme der Kommission „Human-Biomonitoring“ des Umweltbundesamtes. Bundesgesundheitsbl-Gesundheitsforsch-Gesundheitsschutz 2003; 46: $1112-1113$

4 Anonym. Quecksilberintoxikation. Bleichsalbe wegen Aberglaubens angewendet. Deutsches Ärzteblatt 2001; 98: B1997

5 Anonym. Migranten: Quecksilber-Intoxikation, Deutsches Ärzteblatt 98, Ausgabe 37 v. 14. 9. 2001, AKTUELL, Seite A-2305/B-1967/C-1850

6 Dyall-Smith DJ, Scurry JP. Mercury pigmentation and high mercury levels from the use of a cosmetic cream. Med J Aust 1990; 153: 409410

7 Garza-Ocanas L, Torres-Alanis O, Pineyro-Lopez A. Urinary mercury in twelve cases of cutaneous mercurous chloride (calomel) exposure: effect of sodium, 2,3-dimercaptopropane-1-sulfonate (DMPS) therapy. J Toxicol Clin Toxicol 1997; 35: 653 - 655

8 Gonzalez-Ramirez D, Zuniga-Charles M, Narro-Juarez A, Molina-Recio Y, Hurlbut KM, Dart RC, Aposhian HV. DMPS (2,3-dimercaptopropane1 -sulfonate, dimaval) decreases the body burden of mercuryy in humans exposed to mercurous chloride. J Pharmacol Exp Ther 1998; 287: $8-12$

9 Hahn A, Michalak H, Begemann K, Preußner K, Engler A, Rüdiger T, Heinemeyer G, Gundert-Remy $U$. Bundesinstitut für Risikobewertung
(BfR), Bericht der „Zentralen Erfassungsstelle für Vergiftungen, gefährliche Stoffe und Zubereitungen, Umweltmedizin“ im BfR 2002: $52-55$

10 Jovanovic S, Maisner V, Horras-Hun G, Gabrio T, Schwenk M. Poisoning of a family by a mercury-containing ointment. Gesundheitswesen 1997; 59: 405-408

11 Kern F, Roberts N, Ostlere L, Langtry J, Staughton RC. Ammoniated mercury ointment as a cause of peripheral neuropathy. Dermatologica 1991; 183: 280-282

12 Mahé A, Ly F, Aymard G, Dangou JM. Skin diseases associated with the cosmetic use of bleaching products in women from Dakar, Senegal. Br J Dermatol 2003; 148: $493-500$

13 Morton J, Mason HJ, Ritchie KA, White M. Comparison of hair, nails and urine for biological monitoring of low level inorganic mercury exposure in dental workers. Biomarkers 2004; 9: 47-55

14 Otto M, Ahlemeyer C, Tasche H, v. Mühlendahl KE. Endemische Quecksilberbelastung durch eine Bleichsalbe bei Balkanflüchtlingen. Gesundheitswesen 1994; 56: 686-689

15 Pelclová D, Lukas E, Urban J, Preiss J, Rysavá R, Lebenhart P, Okrouhlik B, Fenclocá Z, Lebedová J, Stejskalová A, Ridzon P. Mercury intoxication from skin ointment containing mercuric ammonium chloride. Int Arch Occup Environmental Health 2002; 75: 54-59

16 Schweinsberg $F$. Bedeutung von Quecksilber in der Umweltmedizin eine Übersicht. Umweltmed Forsch Prax 2002; 7: 263-278

17 von Mühlendahl KE. Die Feer'sche Krankheit. Monatsschrift für Kinderheilkunde 1991; 139: 224-227

18 von Mühlendahl KE, Oberdisse U, Bunjes R, Ritter S. Vergiftungen im Kindesalter. 4. Auflage. Stuttgart: Ferdinand Enke Verlag, 2003: 201 295 